\title{
Characterization of Monoclonal Antibody LpMab-3 Recognizing Sialylated Glycopeptide of Podoplanin
}

\author{
Hiroharu Oki, ${ }^{1,2 *}$ Satoshi Ogasawara, ${ }^{1 *}$ Mika Kato Kaneko,, ${ }^{1 *}$ Michiaki Takagi, \\ Masanori Yamauchi, ${ }^{3}$ and Yukinari Kato ${ }^{1}$
}

Podoplanin (PDPN/Aggrus/T1 $\alpha /$ gp36/OTS-8), a type I transmembrane sialoglycoprotein, is involved in platelet aggregation, cell invasion, and cancer metastasis. Podoplanin expression in cancer cells or cancer-associated fibroblasts was reported to be involved in poor prognosis of several cancers. Furthermore, podoplanin is expressed in lymphatic endothelial cells or lung type I alveolar cells. Although many anti-podoplanin monoclonal antibodies (MAbs), such as NZ-1 and D2-40, have been established, almost all anti-podoplanin MAbs are produced against a platelet aggregation-inducing (PLAG) domain. In this study, we produced and characterized a novel anti-podoplanin monoclonal antibody, LpMab-3, the epitope of which is a sialylated glycopeptide of podoplanin. We identified the minimum epitope of LpMab-3 as Thr76-Glu81 of human podoplanin, which is different from PLAG domain, using Western blot analysis and flow cytometry. Immunohistochemical analysis showed that LpMab-3 is useful for detecting lung type I alveolar cells and lymphatic endothelial cells. Because LpMab-3 detects only sialylated podoplanin, it could be useful for uncovering the physiological function of sialylated human podoplanin.

\section{Introduction}

$\mathbf{P}$ ODOPLANIN (PDPN/Aggrus/T1 $\alpha / g p 36 / O T S-8)$ is a platelet aggregation-inducing mucin-type glycoprotein that is involved in cancer metastasis. ${ }^{(1,2)}$ Expression of podoplanin has been reported in many cancers including malignant gliomas, lung cancer, esophageal cancer, malignant mesotheliomas, testicular tumors, bladder cancer, and osteosarcoma. ${ }^{(1,3-14)}$ Moreover, podoplanin expression in cancerassociated fibroblasts (CAFs) was reported to be involved in poor prognosis of several cancers. ${ }^{(15-20)} \mathrm{We}$ previously identified C-type lectin-like receptor-2 (CLEC-2) as an endogenous receptor of podoplanin ${ }^{(21,22)}$ and recently performed comparative crystallographic studies of podoplanin in complex with CLEC-2. ${ }^{(23)}$ The interaction with CLEC-2 was mainly observed at Glu47 and Asp48 in the PLAG3 domain and the $\alpha 2-6$ linked sialic acid at Thr52 of podoplanin.

Anti-podoplanin MAbs with high sensitivity and specificity are necessary to analyze the physiological function of podoplanin in normal tissues and cancers. Although many anti-podoplanin MAbs have been produced, almost all anti-podoplanin MAbs react with a platelet aggregation-inducing (PLAG) domain of human podoplanin. ${ }^{(7,24-28)}$ Rabbit polyclonal antibodies pro- duced by immunizing recombinant rat podoplanin also recognize PLAG domains, which were shown to be immunodominant antigenic sites. ${ }^{(29)}$ We recently established the platform to produce cancer-specific MAbs (CasMabs). ${ }^{(30)}$ In this study, we produced and characterized a novel anti-podoplanin monoclonal antibody, LpMab-3, one of non-CasMabs.

\section{Materials and Methods}

\section{Cell lines and tissues}

Chinese hamster ovary (CHO)-K1, glycan-deficient $\mathrm{CHO}$ cell lines (Lec1, Lec2, and Lec8), LN229, NCI-H226, and P3U1 were purchased from the American Type Culture Collection (ATCC, Manassas, VA). Human lymphatic endothelial cells (LEC) were obtained from Cambrex (Walkersville, MD). The human glioblastoma cell line LN319 was donated by Dr. Webster K. Cavenee (Ludwig Institute for Cancer Research, San Diego, CA). CHO-K1, Lec1, Lec2, Lec8, and LN229 were transfected with human podoplanin plasmids (CHO/hPDPN, Lec1/hPDPN, Lec2/hPDPN, Lec8/ hPDPN, and LN229/hPDPN) using Lipofectamine 2000 (Life Technologies, Carlsbad, CA) according to the manufacturer's instructions. ${ }^{(30)} \mathrm{CHO}-\mathrm{K} 1$, Lec1, Lec2, Lec8, NCI-H226, and

\footnotetext{
${ }^{1}$ Department of Regional Innovation, ${ }^{3}$ Department of Anesthesiology and Perioperative Medicine, Tohoku University Graduate School of Medicine, Sendai, Miyagi, Japan.

${ }^{2}$ Department of Orthopaedic Surgery, Yamagata University Faculty of Medicine, Yamagata, Japan.

*These authors contributed equally to this work.

(c) Hiroharu Oki et al., 2015; Published by Mary Ann Liebert, Inc. This Open Access article is distributed under the terms of the Creative Commons License (http://creativecommons.org/licenses/by/4.0), which permits unrestricted use, distribution, and reproduction in any medium, provided the original work is properly credited.
} 
P3U1 were cultured in RPMI 1640 medium (Wako Pure Chemical Industries, Osaka, Japan), and LN229 and LN319 were cultured in Dulbecco's Modified Eagle's Medium (DMEM) medium (Wako Pure Chemical Industries), supplemented with $10 \%$ heat-inactivated fetal bovine serum (FBS; Life Technologies), $2 \mathrm{mM}$ L-glutamine (Life Technologies), $100 \mathrm{U} / \mathrm{mL}$ of penicillin, and $100 \mu \mathrm{g} / \mathrm{mL}$ of streptomycin (Life Technologies) at $37^{\circ} \mathrm{C}$ in a humidified atmosphere of $5 \% \mathrm{CO}_{2}$ and $95 \%$ air. L-proline $(0.04 \mathrm{mg} / \mathrm{mL})$ was added for Lec 1 , Lec2, and Lec8. LEC was cultured in endothelial cell medium EGM-2MV supplemented with 5\% FBS (Cambrex). Tissue microarrays were purchased from Cybrdi (Frederick, MD).

\section{Antibodies}

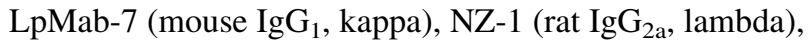
r2336 (rabbit polyclonal), and RMab-3 (mouse $\mathrm{IgG}_{1}$, kappa) were developed previously in our laboratories. (7,24,30,31) $^{2}$ Anti-FLAG tag MAb (1E6) and anti- $\beta$-actin MAb (AC15) were purchased from Wako Pure Chemical Industries and Sigma-Aldrich (St. Louis, MO), respectively.

\section{Hybridoma production}

BALB/c mice were immunized by intraperitoneal (i.p.) injection of $1 \times 10^{8} \mathrm{LN} 229 / \mathrm{hPDPN}$ cells together with Imject Alum (Thermo Fisher Scientific, Waltham, MA). After several additional immunizations, a booster injection was given i.p. 2 days before spleen cells were harvested. The spleen cells were fused with P3U1 cells using GenomONE-CF (Ishihara Sangyo Kaisha, Osaka, Japan). The hybridomas were grown in RPMI medium with hypoxanthine, aminopterin, and thymidine selection medium supplement (Life Technologies). The culture supernatants were screened using enzyme-linked immunosorbent assay (ELISA) for binding to recombinant human podoplanin purified from LN229/ hPDPN cells. Next, flow cytometry was performed against LN229/hPDPN and LN229 cells.

\section{Enzyme-linked immunosorbent assay}

Purified proteins were immobilized on Nunc Maxisorp 96well immunoplates (Thermo Fisher Scientific) at $1 \mu \mathrm{g} / \mathrm{mL}$ for $30 \mathrm{~min} .^{(30)}$ After blocking with SuperBlock T20 (PBS) blocking buffer (Thermo Fisher Scientific), the plates were incubated with culture supernatant or purified MAbs ( $1 \mu \mathrm{g} / \mathrm{mL})$ followed by 1:1000 diluted peroxidase-conjugated anti-mouse IgG (Dako, Glostrup, Denmark). The enzymatic reaction was conducted with a 1-Step Ultra TMB-ELISA (Thermo Fisher Scientific). The optical density was measured at $655 \mathrm{~nm}$ using an iMark microplate reader (Bio-Rad Laboratories, Philadelphia, PA). These reactions were performed with a volume of $50 \mu \mathrm{L}$ at $37^{\circ} \mathrm{C}$.

\section{Production of podoplanin mutants}

The amplified human podoplanin cDNA was subcloned into a pcDNA3 vector (Life Technologies) and a FLAG epitope tag was added at the C-terminus. Substitution of amino acids to alanine in podoplanin was performed using a QuikChange Lightning site-directed mutagenesis kit (Agilent Technologies, Santa Clara, CA). ${ }^{(30,32)}$ CHO-K1 cells were transfected with the plasmids using a Gene Pulser Xcell electroporation system (Bio-Rad Laboratories).

\section{Flow cytometry}

Cell lines were harvested by brief exposure to $0.25 \%$ Trypsin/1 mM EDTA (Wako Pure Chemical Industries). ${ }^{(22)}$ After washing with phosphate-buffered saline (PBS), the cells were treated with primary antibodies $(1 \mu \mathrm{g} / \mathrm{mL})$ for $30 \mathrm{~min}$ at $4^{\circ} \mathrm{C}$, followed by treatment with Oregon greenconjugated anti-mouse IgG (Life Technologies), Alexa Fluor 488 conjugated anti-mouse IgG (Cell Signaling Technology, Danvers, MA), or Alexa Fluor 488 conjugated anti-rat IgG (Cell Signaling Technology). Fluorescence data were collected using a FACS Calibur flow cytometer (BD Biosciences, Braintree, MA) or a Cell Analyzer EC800 (Sony, Tokyo, Japan).

\section{Western blot analyses}

Cell lysates $(10 \mu \mathrm{g})$ were boiled in SDS sample buffer (Nacalai Tesque, Kyoto, Japan). ${ }^{(33)}$ The proteins were electrophoresed on 5-20\% polyacrylamide gels (Wako Pure Chemical Industries) and were transferred onto a PVDF membrane (EMD Millipore, Billerica, MA). After blocking with SuperBlock T20 (PBS) Blocking Buffer, the membrane was incubated with primary antibodies $(1 \mu \mathrm{g} / \mathrm{mL})$, and then with peroxidase-conjugated secondary antibodies (Dako, Glostrup, Denmark; 1:1000 diluted), and developed with the ECL-plus reagent (Thermo Fisher Scientific) using a SayacaImager (DRC, Tokyo, Japan).

\section{Immunohistochemical analyses}

Four- $\mu \mathrm{m}$-thick histologic sections were deparaffinized in xylene and rehydrated. Then they were autoclaved in citrate buffer (pH 6.0; Dako) for $20 \mathrm{~min}$. Sections were incubated with $5 \mu \mathrm{g} / \mathrm{mL}$ of LpMab-3 overnight at $4^{\circ} \mathrm{C}$ followed by treatment with an Envision + kit (Dako). Color was developed using 3,3-diaminobenzidine tetrahydrochloride (DAB; Dako) for $10 \mathrm{~min}$, and the sections were counterstained with hematoxylin (Wako Pure Chemical Industries).

\section{Affinity determination by surface plasmon resonance}

To determine the affinity, recombinant podoplanin-Fc was immobilized on the surface of chips for analysis using the BIAcore 3000 system (GE Healthcare, Piscataway, NJ). The running buffer was $10 \mathrm{mM}$ HEPES, $150 \mathrm{mM} \mathrm{NaCl}$, and $0.005 \%$ v/v Surfactant P20 (BR-1003-68, pH 7.4; GE Healthcare). LpMab-3 was passed over the biosensor chip, and the affinity rate constants (association rate constant, $k_{\text {assoc }}$, and disassociation rate constant, $k_{\text {diss }}$ ) were determined by nonlinear curve-fitting using the Langmuir one-site binding model of the BIAevaluation software (GE Healthcare). The affinity constant $\left(K_{\mathrm{A}}\right)$ at equilibrium was calculated as $K_{\mathrm{A}}=k_{\text {assoc }} / k_{\text {diss }}$, and the dissociate constant $\left(K_{\mathrm{D}}\right)$ was determined as $1 / K_{\mathrm{A}}$.

\section{Results}

Production and characterization of novel anti-podoplanin monoclonal antibody LpMab-3

To develop novel anti-podoplanin MAbs, we immunized mice with LN229/hPDPN cells. The culture supernatants were screened using ELISA for binding to recombinant human podoplanin purified from LN229/hPDPN cells. After 
limiting the dilution of the hybridomas, LpMab-3 ( $\operatorname{IgG}_{1}$, kappa) was established. LpMab-3 reacted with LN229/ hPDPN, not with LN229, a podoplanin-negative cell line (Fig. 1A). Furthermore, LpMab-3 detected endogenous podoplanin, which is expressed in LN319 (a glioblastoma cell line), a lymphatic endothelial cell (LEC), and NCI-H226 (a malignant mesothelioma cell line) (Fig. 1B). We next performed flow cytometric analyses using LpMab-3 against several glycan-deficient podoplanin transfectants (Fig. 1C). LpMab-7, which was used as a positive control, reacted with
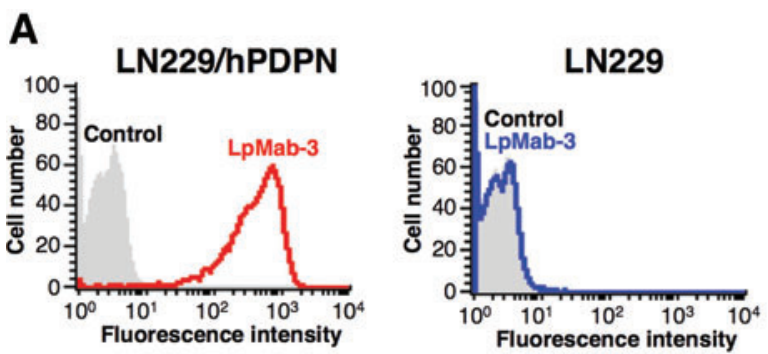

B
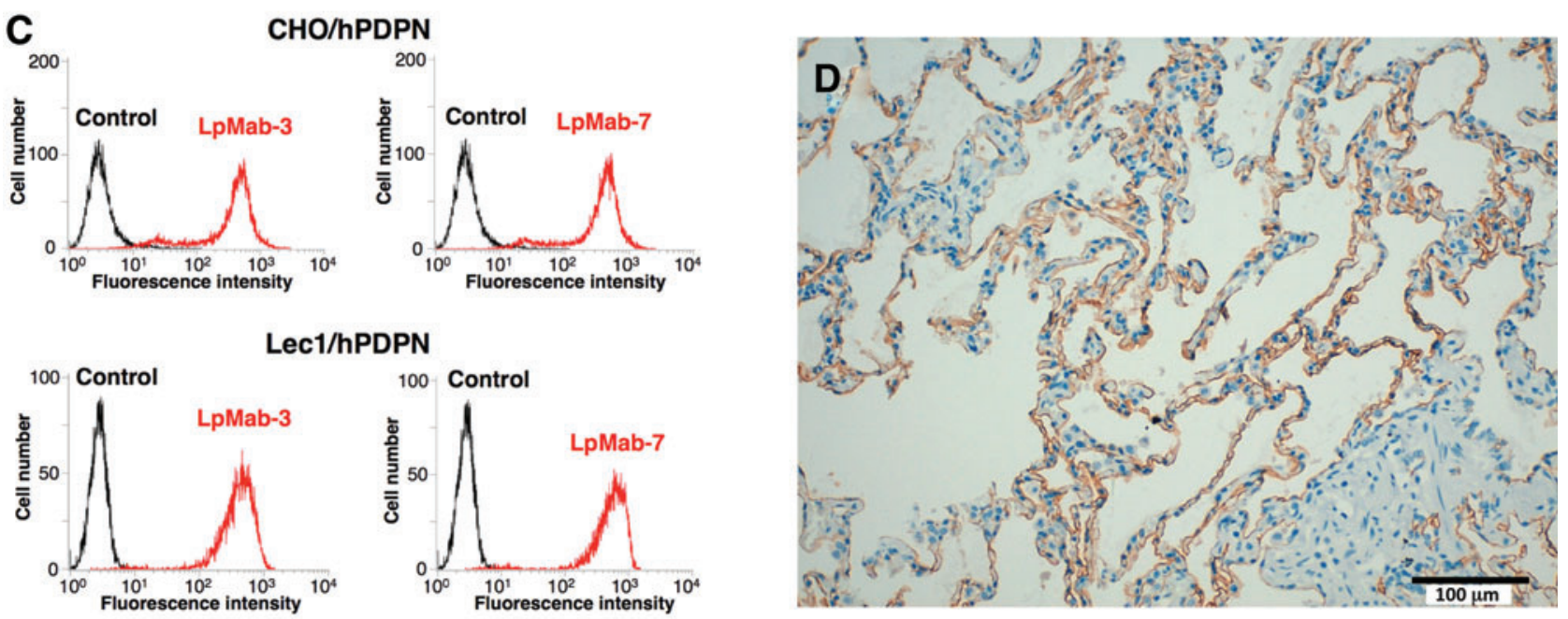

\section{Lec2/hPDPN}
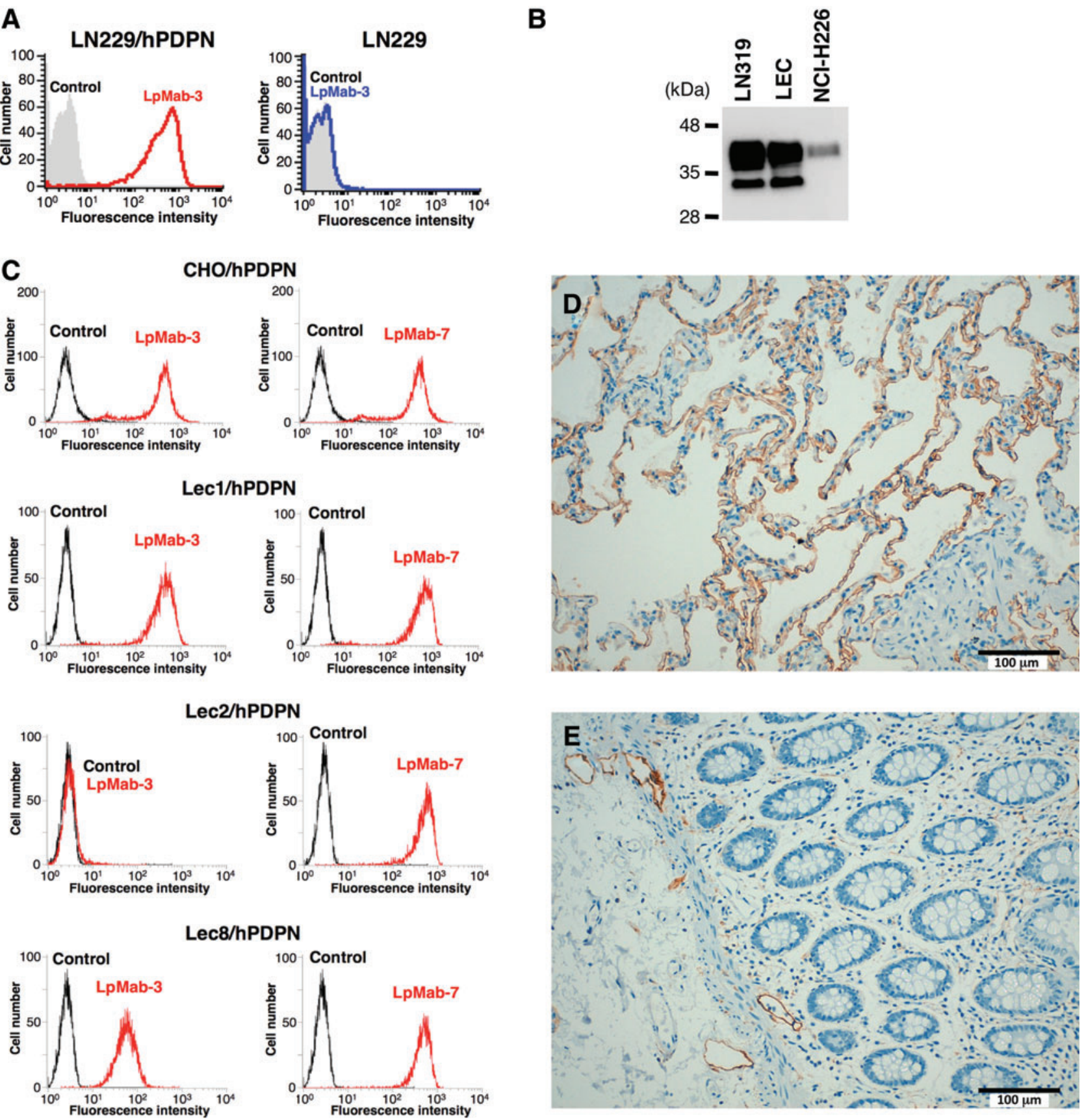

FIG. 1. (A) Flow cytometric analysis by LpMab-3 against LN229/hPDPN and LN229. Cell lines were treated with LpMab-3 $(1 \mu \mathrm{g} / \mathrm{mL})$ for $30 \mathrm{~min}$ at $4{ }^{\circ} \mathrm{C}$, followed by treatment with Oregon green-conjugated anti-mouse IgG. Fluorescence data were collected using a FACS Calibur flow cytometer. (B) Western blot analysis by LpMab-3. Total cell lysate were electrophoresed on 5-20\% polyacrylamide gels and transferred onto a PVDF membrane. After blocking, the membrane was incubated with $1 \mu \mathrm{g} / \mathrm{mL}$ of LpMab-3 and then with peroxidase-conjugated anti-mouse IgG; the membrane was detected using a SayacaImager. (C) Flow cytometric analysis by LpMab-3 and LpMab-7 against glycan-deficient podoplanin-expressing CHO cell lines. Cell lines were treated with LpMab-3 and LpMab-7 $(1 \mu \mathrm{g} / \mathrm{mL})$ for 30 min at $4{ }^{\circ} \mathrm{C}$, followed by treatment with Alexa Fluor 488 conjugated anti-mouse IgG. Fluorescence data were collected using a Cell Analyzer EC800. (D, E) Immunohistochemical analysis against normal tissues using LpMab-3. Sections of normal lung (D) and normal colon (E) were incubated with $5 \mu \mathrm{g} / \mathrm{mL}$ of LpMab-3, followed by Envision + kit. Color was developed using DAB and counterstained with hematoxylin. 
all podoplanin transfectants. In contrast, LpMab-3 did not react with Lec2/hPDPN (sialic acid-deficient), although it reacted with $\mathrm{CHO} / \mathrm{hPDPN}$, Lec1/hPDPN ( $N$-glycan deficient), or Lec8/hPDPN ( $O$-glycan deficient) cells, indicating that LpMab-3 recognizes sialylated podoplanin.

We next performed a kinetic analysis of the interaction of LpMab-3 with a recombinant podoplanin using surface plasmon resonance (BIAcore). Determination of the association and dissociation rates from the sensorgrams revealed that $k_{\text {assoc }}$ of $1.12 \times 10^{4}(\mathrm{~mol} / \mathrm{L}-\mathrm{s})^{-1}$ and $k_{\text {diss }}$ of $9.49 \times 10^{-}{ }^{4} \mathrm{~s}^{-1}$. The $K_{\mathrm{A}}$ at binding equilibrium, calculated as $K_{\mathrm{A}}=k_{\text {assoc }} / k_{\text {diss }}$, was $1.18 \times 10^{7}(\mathrm{~mol} / \mathrm{L})^{-1}, K_{\mathrm{D}}=1 / K_{\mathrm{A}}=8.5 \times 10^{8} \mathrm{M}$. The affinity of LpMab-3 calculated by BIAcore is about 200 times lower than that of NZ-1 $\left(K_{\mathrm{D}}: 4.0 \times 10-{ }^{10} \mathrm{M}\right){ }^{(34)}$

\section{Immunohistochemical analysis against podoplanin- expressing normal tissues using LpMab-3}

We investigated the podoplanin expression in normal lung and colon. As shown in Figure 1D, LpMab-3 detected type I alveolar cells. In our previous study, NZ-1 could not detect type I alveolar cells in immunohistochemistry ${ }^{(10)}$; therefore, LpMab-3 is more useful for detecting type I alveolar cells compared with previous anti-podoplanin MAbs. LpMab-3 also detects lymphatic endothelial cells of normal colon (Fig. 1E). Taken together, LpMab-3 is useful for immunohistochemistry using paraffin-embedded tissues.

\section{Epitope mapping by Western blot analysis and flow cytometry}

To determine the LpMab-3 epitope, we first performed Western blot analysis. LpMab-3 reaction was lost in point mutations of 76-81 amino acids (Fig. 2A). This epitope includes Thr76, the only Ser/Thr residue, indicating that Thr76 is sialylated and is essential for LpMab-3 recognition. In contrast, LpMab-7 reaction was lost in point mutations of 79-83 amino acids. Interestingly, 79-81 amino acids are included as epitopes of both LpMab-3 and LpMab-7. Furthermore, both LpMab-3 and LpMab-7 detects two bands (40 kDa and $30 \mathrm{kDa}$; glycosylated podoplanin), whereas NZ1 (a rat anti-PLAG domain MAb) and r2336 (a rabbit antiN-terminus of podoplanin polyclonal antibody [pAb]) detect only one band ( $40 \mathrm{kDa})$. Anti-FLAG tag MAb detects both two bands of glycosylated podoplanin; therefore, both LpMab-3 and LpMab-7 are more sensitive against podoplanin compared with anti-N-terminus antibodies (NZ-1 and r2336). LpMab-7 and anti-FLAG tag MAbs also detected non-glycosylated podoplanin $(25 \mathrm{kDa})$ of several podoplanin point mutants.

We next performed flow cytometric analysis using LpMab-3 and NZ-1 MAbs against the same point mutants of podoplanin. The results revealed that $\mathrm{LpMab}-3$ did not react with R79A, I80A, and E81A, and weakly reacted with T76A, G77A, and I78A (Fig. 2B), indicating that TGIRIE sequence is the minimum epitope, and Arg79, Ile80, and Glu81 are much more critical residues for LpMab-3 epitopes (Fig. 2C).

\section{Discussion}

Podoplanin is expressed in normal tissues such as lymphatic endothelial cells, lung type I alveolar cells, epidermal keratinocytes, kidney podocytes, and fibroblastic reticular cells (FRCs) of lymph nodes. ${ }^{(35,36)}$ Recently, several physiological functions of podoplanin have been reported. The activation of CLEC-2 by podoplanin (the signal from podoplanin to CLEC-2) rearranges the actin cytoskeleton in dendritic cells to promote efficient motility along stromal surfaces. ${ }^{(37)}$ In contrast, the signal from CLEC-2 to podoplanin controls the contractility of FRCs and lymph node microarchitecture. ${ }^{(38)}$ The physical elasticity of lymph nodes is maintained by podoplanin of stromal FRCs and its modulation by CLEC-2 of dendritic cells. ${ }^{(39)}$ Although we have shown that podoplanin possesses platelet-aggregating activity via CLEC-2 in cancer models, podoplanin-CLEC-2 interaction is also important for embryonic blood-lymphatic vascular separation using platelet aggregation. ${ }^{(1,2,21,22,40-42)}$ The local sphingosine-1-phosphate release after podoplaninCLEC-2-mediated platelet activation is critical for the integrity of high endothelial venules during immune responses. ${ }^{(43)}$ Furthermore, the development of ectopic lymphoid follicles is dependent on Th17-expressing podoplanin. ${ }^{(44)}$ Taken together, the reciprocal interaction between podoplanin and CLEC-2 is important in many physiological functions. Therefore, development of novel anti-podoplanin MAbs, the epitopes of which are different, is still important.

LpMab-3 possesses a unique epitope that is completely different from that previously reported for anti-podoplanin MAbs such as NZ-1 and D2-40. The epitope is similar to that of LpMab-7; however, LpMab-3 needs sialylation of Thr76. Because only $\alpha 2-6$ linked sialic acid was attached to podoplanin on Lec8/hPDPN, ${ }^{(40)}$ LpMab-3 epitope may include $\alpha 2-6$ linked sialic acid, not $\alpha 2-3$ linked sialic acid. Therefore, LpMab-3 is useful for distinguishing Thr76sialylated from Thr76-nonsialylated podoplanin. However, the binding affinity of LpMab-3 was shown to be lower than that of NZ-1. Because the binding affinity of antibodies is critical for antibody-based cancer therapy, affinity maturation of LpMab-3 should be considered in the future. Using the CasMab method, we can obtain not only cancerspecific MAbs (CasMabs) but also non-CasMabs such LpMab-3 and LpMab-7. Of interest, non-CasMabs, such as LpMab-3, also include the glycan within those epitopes. ${ }^{(30)}$

FIG. 2. Epitope mapping of LpMab-7 by Western blot analysis and flow cytometry. (A) Western blotting by LpMab-3, LpMab-7, NZ-1, r2336, 1E6, RMab-3 ( $\alpha$-IDH1), and AC-15 ( $\alpha$ - $\beta$-actin). Total cell lysate were electrophoresed on 5-20\% polyacrylamide gels and transferred onto a PVDF membrane. After blocking, the membrane was incubated with $1 \mu \mathrm{g} / \mathrm{mL}$ of primary antibodies and then with peroxidase-conjugated secondary antibodies; the membrane was detected using a SayacaImager. Blue arrow, $40 \mathrm{kDa}$ band (glycosylated); red arrow, $30 \mathrm{kDa}$ band (glycosylated); black arrow, $25 \mathrm{kDa}$ band (nonglycosylated). (B) Point mutants of human podoplanin were treated with NZ-1 and LpMab-3 (1 $\mu \mathrm{g} / \mathrm{mL})$ for $30 \mathrm{~min}$ at $4{ }^{\circ} \mathrm{C}$, followed by treatment with Alexa Fluor 488 conjugated anti-rat IgG and anti-mouse IgG, respectively. Fluorescence data were collected using a Cell Analyzer EC800. (C) TGIRIE sequence and $\alpha 2-6$ linked sialic acid are the critical epitope of LpMab-3. 
A

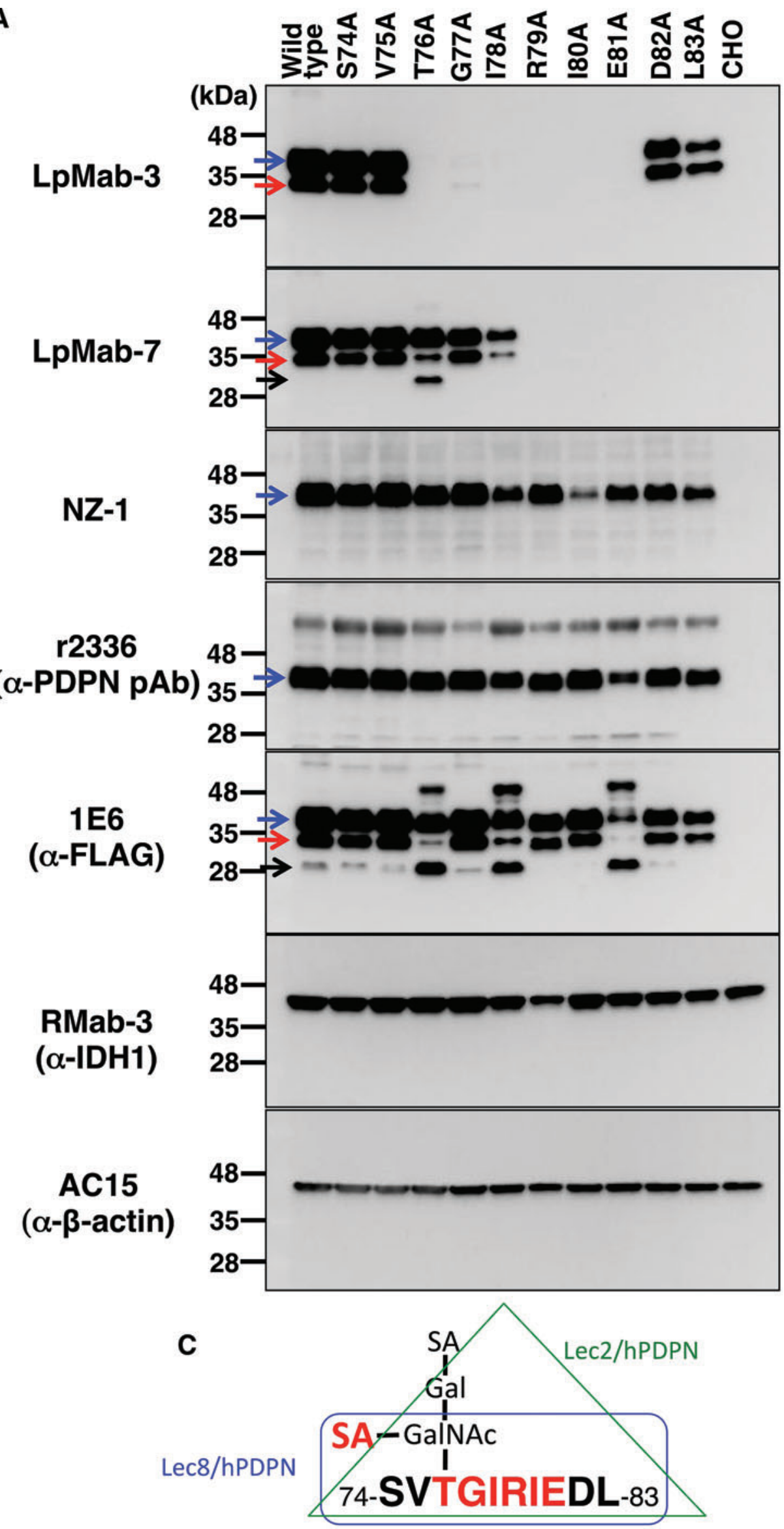

B
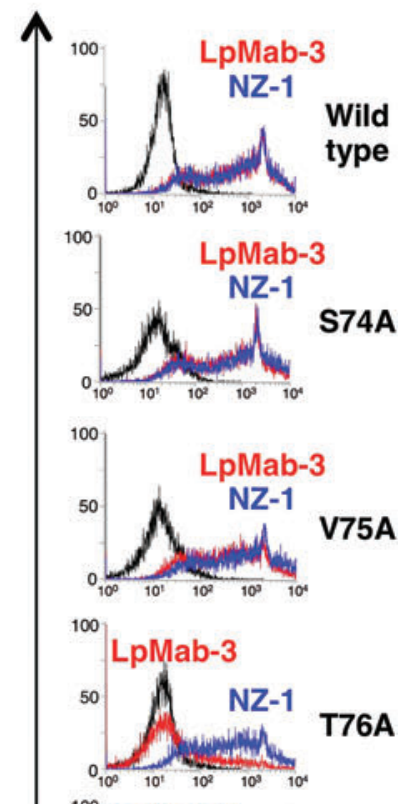

${ }^{100}$ LpMab-3
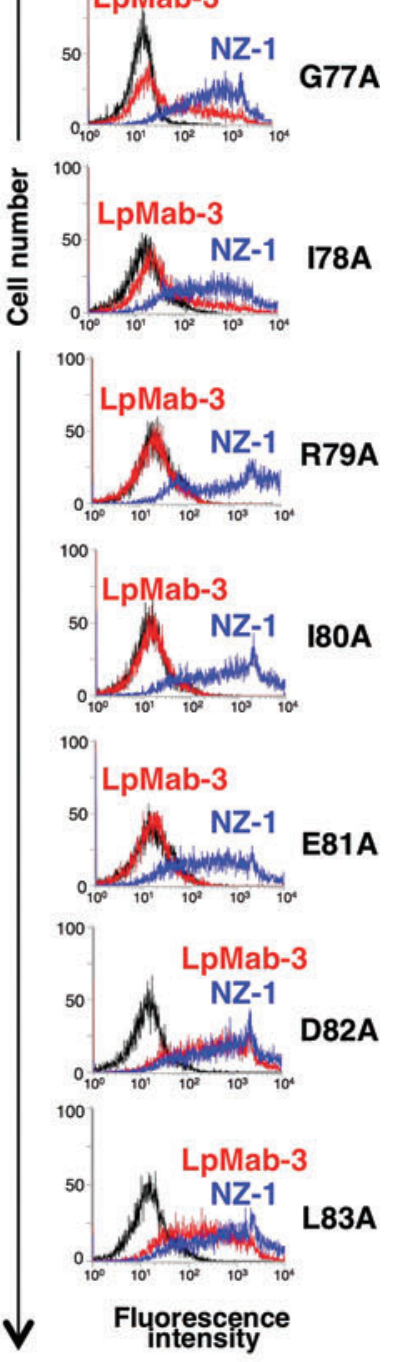
Although antibody-dependent cellular cytotoxicity (ADCC) and complement-dependent cytotoxicity (CDC) activities are very important for an antibody-based molecular targeting therapy, we could not investigate these activities because the subclass of $\mathrm{LpMab}-3$ is mouse $\operatorname{IgG}_{1}$. The conversion of subclass into human $\mathrm{IgG}_{1}$ or mouse $\mathrm{IgG}_{2 \mathrm{a}}$ is necessary to demonstrate $\mathrm{ADCC} / \mathrm{CDC}$ activities.

\section{Acknowledgments}

We thank Takuro Nakamura, Noriko Saidoh, Kanae Yoshida, Xing Liu, and Yuta Tsujimoto for their excellent technical assistance. This work was supported in part by the Platform for Drug Discovery, Informatics, and Structural Life Science (PDIS) from the Ministry of Education, Culture, Sports, Science and Technology (MEXT) of Japan (Y.K.); by the Basic Science and Platform Technology Program for Innovative Biological Medicine from MEXT of Japan (Y.K.); by the Regional Innovation Strategy Support Program from MEXT of Japan (Y.K.); and by a Grant-in-Aid for Scientific Research (M.K.K. and Y.K.) and a Grant-in-Aid for Young Scientists (S.O.) from MEXT of Japan.

\section{Author Disclosure Statement}

The authors have no financial interests to disclose.

\section{References}

1. Kato Y, Fujita N, Kunita A, Sato S, Kaneko M, Osawa M, and Tsuruo T: Molecular identification of Aggrus/T1alpha as a platelet aggregation-inducing factor expressed in colorectal tumors. J Biol Chem 2003;278:51599-51605.

2. Kaneko MK, Kato Y, Kitano T, and Osawa M: Conservation of a platelet activating domain of Aggrus/podoplanin as a platelet aggregation-inducing factor. Gene 2006;378:52-57.

3. Kato Y, Sasagawa I, Kaneko M, Osawa M, Fujita N, and Tsuruo T: Aggrus: a diagnostic marker that distinguishes seminoma from embryonal carcinoma in testicular germ cell tumors. Oncogene 2004;23:8552-8556.

4. Martin-Villar E, Scholl FG, Gamallo C, Yurrita MM, Munoz-Guerra M, Cruces J, and Quintanilla M: Characterization of human PA2.26 antigen (T1alpha-2, podoplanin), a small membrane mucin induced in oral squamous cell carcinomas. Int J Cancer 2005;113:899-910.

5. Kato Y, Kaneko M, Sata M, Fujita N, Tsuruo T, and Osawa M: Enhanced expression of Aggrus (T1alpha/podoplanin), a platelet-aggregation-inducing factor in lung squamous cell carcinoma. Tumor Biol 2005;26:195-200.

6. Yuan P, Temam S, El-Naggar A, Zhou X, Liu D, Lee J, and Mao L: Overexpression of podoplanin in oral cancer and its association with poor clinical outcome. Cancer 2006;107: 563-569.

7. Kato Y, Kaneko MK, Kuno A, Uchiyama N, Amano K, Chiba Y, Hasegawa Y, Hirabayashi J, Narimatsu H, Mishima $\mathrm{K}$, and Osawa M: Inhibition of tumor cell-induced platelet aggregation using a novel anti-podoplanin antibody reacting with its platelet-aggregation-stimulating domain. Biochem Biophys Res Commun 2006;349:1301-1307.

8. Mishima K, Kato Y, Kaneko MK, Nakazawa Y, Kunita A, Fujita N, Tsuruo T, Nishikawa R, Hirose T, and Matsutani M: Podoplanin expression in primary central nervous system germ cell tumors: a useful histological marker for the diagnosis of germinoma. Acta Neuropathol 2006;111:563-568.
9. Mishima K, Kato Y, Kaneko MK, Nishikawa R, Hirose T, and Matsutani M: Increased expression of podoplanin in malignant astrocytic tumors as a novel molecular marker of malignant progression. Acta Neuropathol 2006;111: 483-488.

10. Abe S, Morita Y, Kaneko MK, Hanibuchi M, Tsujimoto Y, Goto H, Kakiuchi S, Aono Y, Huang J, Sato S, Kishuku M, Taniguchi Y, Azuma M, Kawazoe K, Sekido Y, Yano S, Akiyama S, Sone S, Minakuchi K, Kato Y, and Nishioka Y: A novel targeting therapy of malignant mesothelioma using anti-podoplanin antibody. J Immunol 2013;190:6239-6249.

11. Takagi S, Oh-hara T, Sato S, Gong B, Takami M, and Fujita N: Expression of Aggrus/podoplanin in bladder cancer and its role in pulmonary metastasis. Int $\mathrm{J}$ Cancer 2014;134:2605-2614.

12. Kunita A, Kashima TG, Ohazama A, Grigoriadis AE, and Fukayama M: Podoplanin is regulated by AP-1 and promotes platelet aggregation and cell migration in osteosarcoma. Am J Pathol 2011;179:1041-1049.

13. Chandramohan V, Bao X, Kato Kaneko M, Kato Y, Keir ST, Szafranski SE, Kuan CT, Pastan IH, and Bigner DD: Recombinant anti-podoplanin (NZ-1) immunotoxin for the treatment of malignant brain tumors. Int J Cancer 2013; 132:2339-2348.

14. Kato Y, Vaidyanathan G, Kaneko MK, Mishima K, Srivastava N, Chandramohan V, Pegram C, Keir ST, Kuan CT, Bigner DD, and Zalutsky MR: Evaluation of antipodoplanin rat monoclonal antibody NZ-1 for targeting malignant gliomas. Nucl Med Biol 2010;37:785-794.

15. Kawase A, Ishii G, Nagai K, Ito T, Nagano T, Murata Y, Hishida T, Nishimura M, Yoshida J, Suzuki K, and Ochiai A: Podoplanin expression by cancer associated fibroblasts predicts poor prognosis of lung adenocarcinoma. Int $\mathbf{J}$ Cancer 2008;123:1053-1059.

16. Hoshino A, Ishii G, Ito T, Aoyagi K, Ohtaki Y, Nagai K, Sasaki H, and Ochiai A: Podoplanin-positive fibroblasts enhance lung adenocarcinoma tumor formation: podoplanin in fibroblast functions for tumor progression. Cancer Res 2011;71:4769-4779.

17. Pula B, Jethon A, Piotrowska A, Gomulkiewicz A, Owczarek T, Calik J, Wojnar A, Witkiewicz W, Rys J, Ugorski M, Dziegiel P, and Podhorska-Okolow M: Podoplanin expression by cancer-associated fibroblasts predicts poor outcome in invasive ductal breast carcinoma. Histopathology 2011;59:1249-1260.

18. Schoppmann SF, Jesch B, Riegler MF, Maroske F, Schwameis K, Jomrich G, and Birner P: Podoplanin expressing cancer associated fibroblasts are associated with unfavourable prognosis in adenocarcinoma of the esophagus. Clin Exp Metastasis 2013;30:441-446.

19. Shindo K, Aishima S, Ohuchida K, Fujiwara K, Fujino M, Mizuuchi Y, Hattori M, Mizumoto K, Tanaka M, and Oda $\mathrm{Y}$ : Podoplanin expression in cancer-associated fibroblasts enhances tumor progression of invasive ductal carcinoma of the pancreas. Mol Cancer 2013;12:168.

20. Inoue H, Tsuchiya H, Miyazaki Y, Kikuchi K, Ide F, Sakashita $\mathrm{H}$, and Kusama $\mathrm{K}$ : Podoplanin expressing cancerassociated fibroblasts in oral cancer. Tumour Biol 2014;35: 11345-11352.

21. Suzuki-Inoue K, Kato Y, Inoue O, Kaneko MK, Mishima K, Yatomi Y, Yamazaki Y, Narimatsu H, and Ozaki Y: Involvement of the snake toxin receptor CLEC-2, in podoplaninmediated platelet activation, by cancer cells. J Biol Chem 2007;282:25993-26001. 
22. Kato Y, Kaneko MK, Kunita A, Ito H, Kameyama A, Ogasawara S, Matsuura N, Hasegawa Y, Suzuki-Inoue K, Inoue O, Ozaki Y, and Narimatsu H: Molecular analysis of the pathophysiological binding of the platelet aggregationinducing factor podoplanin to the C-type lectin-like receptor CLEC-2. Cancer Sci 2008;99:54-61.

23. Nagae M, Morita-Matsumoto K, Kato M, Kaneko MK, Kato Y, and Yamaguchi Y: A platform of C-type lectin-like receptor CLEC-2 for binding o-glycosylated podoplanin and nonglycosylated rhodocytin. Structure 2014;http:// dx.doi.org/10.1016/j.str.2014.09.009.

24. Ogasawara S, Kaneko MK, Price JE, and Kato Y: Characterization of anti-podoplanin monoclonal antibodies: critical epitopes for neutralizing the interaction between podoplanin and CLEC-2. Hybridoma 2008;27:259-267.

25. Takagi S, Sato S, Oh-hara T, Takami M, Koike S, Mishima Y, Hatake K, and Fujita N: Platelets promote tumor growth and metastasis via direct interaction between Aggrus/ podoplanin and CLEC-2. PLoS One 2013;8:e73609.

26. Nakazawa Y, Takagi S, Sato S, Oh-hara T, Koike S, Takami $\mathrm{M}$, Arai $\mathrm{H}$, and Fujita N: Prevention of hematogenous metastasis by neutralizing mice and its chimeric anti-Aggrus/ podoplanin antibodies. Cancer Sci 2011;102:2051-2057.

27. Marks A, Sutherland DR, Bailey D, Iglesias J, Law J, Lei $\mathrm{M}$, Yeger H, Banerjee D, and Baumal R: Characterization and distribution of an oncofetal antigen (M2A antigen) expressed on testicular germ cell tumours. Br J Cancer 1999;80:569-578.

28. Kono T, Shimoda M, Takahashi M, Matsumoto K, Yoshimoto T, Mizutani M, Tabata C, Okoshi K, Wada H, and Kubo H: Immunohistochemical detection of the lymphatic marker podoplanin in diverse types of human cancer cells using a novel antibody. Int J Oncol 2007;31:501-508.

29. Matsui K, Breiteneder-Geleff S, and Kerjaschki D: Epitope-specific antibodies to the $43-\mathrm{kD}$ glomerular membrane protein podoplanin cause proteinuria and rapid flattening of podocytes. J Am Soc Nephrol 1998;9:2013-2026.

30. Kato Y, and Kaneko MK: A cancer-specific monoclonal antibody recognizes the aberrantly glycosylated podoplanin. Sci Rep 2014;4:5924.

31. Kato Y: Specific monoclonal antibodies against IDH1/2 mutations as diagnostic tools for gliomas. Brain Tumor Pathol Oct 2014.

32. Kaneko MK, Tian W, Takano S, Suzuki H, Sawa Y, Hozumi Y, Goto K, Yamazaki K, Kitanaka C, and Kato Y: Establishment of a novel monoclonal antibody SMab-1 specific for IDH1-R132S mutation. Biochem Biophys Res Commun 2011;406:608-613.

33. Kaneko MK, Kunita A, Abe S, Tsujimoto Y, Fukayama M, Goto K, Sawa Y, Nishioka Y, and Kato Y: Chimeric antipodoplanin antibody suppresses tumor metastasis through neutralization and antibody-dependent cellular cytotoxicity. Cancer Sci 2012;103:1913-1919.

34. Fujii Y, Kaneko M, Neyazaki M, Nogi T, Kato Y, and Takagi J: PA tag: a versatile protein tagging system using a super high affinity antibody against a dodecapeptide derived from human podoplanin. Protein Expr Purif 2014;95:240-247.

35. Breiteneder-Geleff S, Matsui K, Soleiman A, Meraner P, Poczewski H, Kalt R, Schaffner G, and Kerjaschki D: Podoplanin, novel 43-kd membrane protein of glomerular epithelial cells, is down-regulated in puromycin nephrosis. Am J Pathol 1997;151:1141-1152.

36. Schacht V, Dadras SS, Johnson LA, Jackson DG, Hong YK, and Detmar M: Up-regulation of the lymphatic marker podoplanin, a mucin-type transmembrane glycoprotein, in human squamous cell carcinomas and germ cell tumors. Am J Pathol 2005;166:913-921.

37. Acton SE, Astarita JL, Malhotra D, Lukacs-Kornek V, Franz B, Hess PR, Jakus Z, Kuligowski M, Fletcher AL, Elpek KG, Bellemare-Pelletier A, Sceats L, Reynoso ED, Gonzalez SF, Graham DB, Chang J, Peters A, Woodruff M, Kim YA, Swat W, Morita T, Kuchroo V, Carroll MC, Kahn ML, Wucherpfennig KW, and Turley SJ: Podoplanin-rich stromal networks induce dendritic cell motility via activation of the C-type lectin receptor CLEC-2. Immunity 2012;37:276-289.

38. Astarita JL, Cremasco V, Fu J, Darnell MC, Peck JR, Nieves-Bonilla JM, Song K, Kondo Y, Woodruff MC, Gogineni A, Onder L, Ludewig B, Weimer RM, Carroll MC, Mooney DJ, Xia L, and Turley SJ: The CLEC-2-podoplanin axis controls the contractility of fibroblastic reticular cells and lymph node microarchitecture. Nat Immunol Jan 2015.

39. Acton SE, Farrugia AJ, Astarita JL, Mourao-Sa D, Jenkins RP, Nye E, Hooper S, van Blijswijk J, Rogers NC, Snelgrove KJ, Rosewell I, Moita LF, Stamp G, Turley SJ, Sahai E, and Reis e Sousa C: Dendritic cells control fibroblastic reticular network tension and lymph node expansion. Nature 2014;514:498-502.

40. Kaneko M, Kato Y, Kunita A, Fujita N, Tsuruo T, and Osawa M: Functional sialylated O-glycan to platelet aggregation on Aggrus (T1alpha/podoplanin) molecules expressed in Chinese Hamster Ovary cells. J Biol Chem 2004;279:38838-38843.

41. Kaneko MK, Kato Y, Kameyama A, Ito H, Kuno A, Hirabayashi J, Kubota T, Amano K, Chiba Y, Hasegawa Y, Sasagawa I, Mishima K, and Narimatsu H: Functional glycosylation of human podoplanin: glycan structure of platelet aggregation-inducing factor. FEBS Lett 2007;581:331-336.

42. Bertozzi CC, Schmaier AA, Mericko P, Hess PR, Zou Z, Chen M, Chen CY, Xu B, Lu MM, Zhou D, Sebzda E, Santore MT, Merianos DJ, Stadtfeld M, Flake AW, Graf T, Skoda R, Maltzman JS, Koretzky GA, and Kahn ML: Platelets regulate lymphatic vascular development through CLEC-2-SLP-76 signaling. Blood 2010;116:661-670.

43. Herzog BH, Fu J, Wilson SJ, Hess PR, Sen A, McDaniel JM, Pan Y, Sheng M, Yago T, Silasi-Mansat R, McGee S, May F, Nieswandt B, Morris AJ, Lupu F, Coughlin SR, McEver RP, Chen H, Kahn ML, and Xia L: Podoplanin maintains high endothelial venule integrity by interacting with platelet CLEC-2. Nature 2013;502:105-109.

44. Peters A, Pitcher LA, Sullivan JM, Mitsdoerffer M, Acton SE, Franz B, Wucherpfennig K, Turley S, Carroll MC, Sobel RA, Bettelli E, and Kuchroo VK: Th17 cells induce ectopic lymphoid follicles in central nervous system tissue inflammation. Immunity 2011;35:986-996.

Address correspondence to: Yukinari Kato Department of Regional Innovation Tohoku University Graduate School of Medicine 2-1 Seiryo-machi, Aoba-ku Sendai, Miyagi 980-8575 Japan

E-mail: yukinarikato@med.tohoku.ac.jp Received: November 4, 2014 Accepted: December 3, 2014 